\title{
Evaluation of Effective Thermal Conductivities of Porous Textile Composites
}

\author{
Blanka Tomková ${ }^{1}$, Michal Šejnoha ${ }^{2,3}$, Jan Novák ${ }^{2,3}$ and Jan Zeman ${ }^{2 *}$ \\ ${ }^{1}$ Department of Textile Materials, Technical University in Liberec \\ Hálkova 6, 46117 Liberec 1, Czech Republic \\ ${ }^{2}$ Department of Mechanics, Faculty of Civil Engineering \\ Czech Technical University in Prague \\ Thákurova 7, 16629 Prague 6, Czech Republic \\ ${ }^{3}$ Centre for Integrated Design of Advances Structures \\ Thákurova 7, 16629 Prague 6, Czech Republic
}

\begin{abstract}
An uncoupled multi-scale homogenization approach is used to estimate the effective thermal conductivities of plain weave $\mathrm{C} / \mathrm{C}$ composites with a high degree of porosity. The geometrical complexity of the material system on individual scales is taken into account through the construction of a suitable representative volume element (RVE), a periodic unit cell, exploiting the information provided by the image analysis of a real composite system on every scale. Two different solution procedures are examined. The first one draws on the classical first order homogenization technique assuming steady state conditions and periodic distribution of the fluctuation part of the temperature field. The second approach is concerned with the solution of a transient flow problem. Although more complex, the latter approach allows for a detailed simulation of heat transfer in the porous system. Effective thermal conductivities of the laminate derived from both approaches through a consistent homogenization on individual scales are then compared with those obtained experimentally. A reasonably close agreement between individual results then promotes the use of the proposed multi-scale computational approach combined with the image analysis of real material systems.
\end{abstract}

Keywords thermal properties, finite element analysis, micro-mechanics, carbon-carbon plain weave composite

Accepted in International Journal for Multiscale Computational Engineering

\footnotetext{
${ }^{*}$ Corresponding author, Tel.: +420-2-2435-4482; fax +420-2-2431-0775, E-mail addresses: tomkova@tulib.cz, sejnom@fsv.cvut.cz, novakj@cml.fsv.cvut.cz, zemanj@cml.fsv.cvut.cz
} 


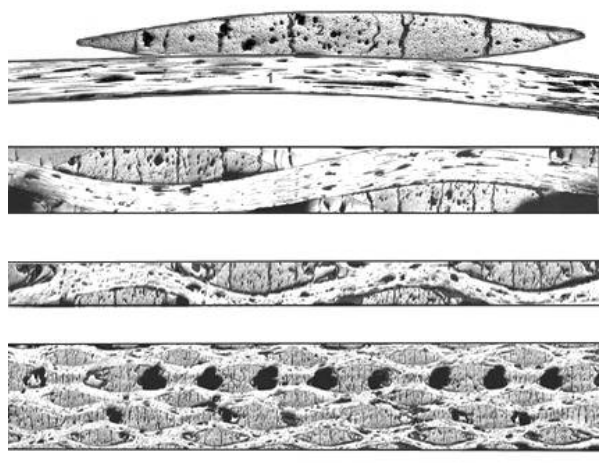

(a)

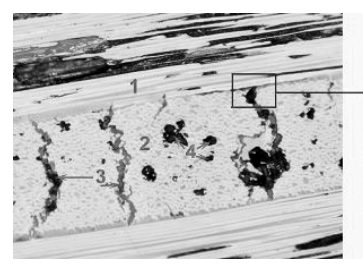

(b)

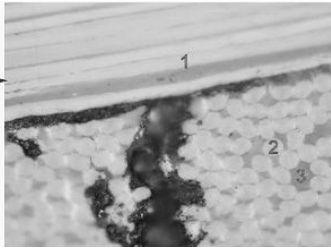

b)

Figure 1: Color images of a real composite system: (a) Scheme of multiscale structural model (from top - transverse and longitudinal view of fiber tow composite, composite unit cell, composite lamina, composite plate), (b) Carbon tow microstructure showing major pores and transverse cracks.

\section{Introduction}

Carbon-carbon $(\mathrm{C} / \mathrm{C})$ plain weave fabric composites belong to an important class of hightemperature material systems. An exceptional thermal stability together with high resistance to thermal shocks or fracture due to rapid and strong changes in temperature have made these materials almost indispensable in a variety of engineering spheres including aeronautics, space and automobile industry. Applications include components in spacecraft protective shields, wing leading edges or parts of jet aircrafts turbine engines.

While their appealing thermal properties such as low coefficients of thermal expansion and high thermal conductivities are known, their prediction from the properties supplied by the manufacturer for individual constituents is far from being trivial since these systems are generally highly complicated. Apart from a characteristic three-dimensional (3D) structure of textile composites the geometrical complexity is further enhanced by the presence of various imperfections in woven path developed during the manufacturing process. A route for incorporating at least the most severe imperfections in predictions of the mechanical properties of these systems has been outlined in [1] in the context of statistically equivalent periodic unit cell. Although properly accounting for three-dimensional effects the resulting representative volume element still suffers from the absence of the porous phase, which in real systems, Fig. 1, may exceed $30 \%$ of the overall volume. As suggested in [2], neglecting the material porosity may severely overestimate the resulting thermal properties of textile composites. In this regard, the procedure introduced in the current paper extends the previous studies, as they either neglect the porous phase [3, 4] or analyze the porosity effects on the level of fibers and tows only [5].

The porosity of $\mathrm{C} / \mathrm{C}$ composites directly arises as a result of the manufacturing process characterized by thermal decomposition and transformation of an initial polymeric precursor into the carbon matrix through several steps of carbonization, re-impregnation and final graphitization. As evident from Fig. 1 the major contribution to the porosity is due to crimp voids and delamination cracks, which are usually classified as inter-tow voids (Fig. 1(a)), as well as due to intra-tow voids represented by pores and transverse cracks developed within the fiber tow composite (Fig. 1(b)). For more details the reader is referred to [6] and references therein.

While simplifying averaging schemes may provide rational micromechanics models for the prediction of effective thermal conductivities [7] at the level of the fiber tow composite in 
Fig. 1(b), the complex mesoscopic structure of woven fabric plotted in Fig. 1(a) calls for considerably more accurate treatment of the actual geometry as already demonstrated in $[1,8]$. In such a case, the image analysis combined with a reliable morphological description of the underlying composite structure then provide a general tool for the determination of what has been termed the statistically equivalent periodic unit cell (SEPUC) introduced by the authors in their previous works on random and imperfect composites $[9,1,8]$. Unlike classical averaging schemes, information on the local fields in representative volume elements formulated on the bases of SEPUCs is derived through a detailed numerical analysis which typically employs the finite element method (FEM). A special treatment of boundary conditions is then needed to establish a link with the first-order homogenization scheme which develops upon the assumption of homogeneous effective (macroscopic) fields [10].

Existence of macroscopically uniform fields (strains or stresses in the case of mechanical problem or temperature gradient and heat flux in the case of heat conduction problem) readily allows for splitting the local fields into macroscopic and fluctuation parts which in view of the solution of heat conduction problem reads

$$
\theta(\boldsymbol{x})=\boldsymbol{H} \cdot \boldsymbol{x}+\theta^{*}(\boldsymbol{x}) \quad \text { or } \quad \theta(\boldsymbol{x})=H_{i} x_{i}+\theta^{*}(\boldsymbol{x}),
$$

where $\boldsymbol{H}$ represents the macroscopically uniform temperature gradient vector and $\theta^{*}(\boldsymbol{x})$ is the fluctuation part of the local temperature $\theta(\boldsymbol{x})$. Following, e.g. [11, 12] the solution of Eq. (1) then turns into the search for $\theta^{*}$ in terms of the applied macroscopic uniform temperature gradient $\boldsymbol{H}$ or the macroscopic uniform heat flux $\boldsymbol{Q}$. Consistency between the macroscopic (homogenized) quantities and volume averages of the corresponding local fields then requires either setting the boundary values of $\theta^{*}$ equal to zero or subjecting the fluctuation part of temperature field to periodic constrains. While both types of boundary conditions are equally applicable ensuring the macroscopic heat flux being equal to the volume averaged microscopic (local) heat flux, the latter conditions will be employed in Section 3 when developing the framework for heat conduction problems as they were shown to provide the best approximation for a fixed RVE size in a purely mechanical analysis, see e.g. $[13,14]$. The need for periodic boundary conditions also arises when departing from asymptotic homogenization, see e.g. [15].

It is also interesting to point out that Eq. (1), when applied to either constrains on $\theta^{*}$, is consistent with so called affine boundary conditions represented in our particular case by homogeneous temperature or flux applied on the outside boundary $\Gamma$ of the RVE as

$$
\begin{array}{llrl}
\bar{\theta}(\boldsymbol{x}) & =\boldsymbol{H} \cdot \boldsymbol{x}, & \bar{q}_{n}(\boldsymbol{x})=\boldsymbol{Q} \cdot \boldsymbol{n}, & \text { on } \Gamma, \\
\bar{\theta}(\boldsymbol{x})=H_{i} x_{i}, & \bar{q}_{n}(\boldsymbol{x})=Q_{i} n_{i}, & \text { on } \Gamma,
\end{array}
$$

where $\boldsymbol{n}$ is the outer normal to $\Gamma$. Although the solution of a steady state heat conduction problem driven by prescribed macroscopic temperature gradient (or boundary temperatures consistent with Eq. (2)1 ), especially if the phase thermal conductivities are temperature independent, provides directly the effective conductivity matrix, it appears useful, particularly for more complex geometries as those in Fig. 1(a), to run the time dependent transient heat conduction problem which allows for extracting considerably more information regarding thermal behavior of the composite material. Quite often, as will also be the case in this study, a commercial code, which does not allow for direct introduction of periodic boundary conditions, is used. In such a case, the boundary conditions given by Eq. (2) prove particularly useful as they naturally ensure that the volume average of microscopic (local) fields (temperature gradient or heat flux) are equal to their macroscopic (prescribed) counterparts $\boldsymbol{H}$ and $\boldsymbol{Q}$ as evident from Eqs. (9) and (11).

To construct a certain periodic unit representing an entire laminate with all relevant geometrical details (distribution fibers within the fiber tow composite, waviness of fiber tow path, 
porosity, etc.) might, however, prove rather impractical particularly from the computational point of view. Instead a so called uncoupled multi-scale approach [16], still at the forefront of material science interest, seems rather attractive allowing us to address the material complexity separately at different levels. Three particular levels of interest can be identified for the textile composite under consideration. Henceforth, they will be referred to as micro-scale (the level of individual fibers within a fiber tow, Fig. 1(b)), meso-scale (the level of a fiber tow composite, Fig. 1(a) $)_{1-3}$ from top to the bottom) and macro-scale (the level of a laminated plate, Fig. 1(a) $)_{4}$, respectively. To estimate a response of a such complex structure it appears reasonable to perform a sequence of uncoupled analyses corresponding to individual scales. For this approach to be successfully utilized it is then viable to establish a link between individual scales. Here, the concept of scale separation plays a crucial role in the sense that the three analyses can be carried out independently such that output from one is used as an input to the other in terms of volume averages of the local fields while taking into account the boundary conditions mentioned in the above paragraphs. Such an approach is also adopted in the present study leading to a consistent search for the effective (macroscopic) thermal conductivities of a plain weave highly porous fabric composite.

The paper is organized as follows. Following the introductory part our attention is paid in Section 2 to the formulation of various unit cells associated with individual scales. Theoretical formulation of the homogenization procedure for a steady state heat conduction problem is outlined in Section 3. Section 4 then illustrates the efficiency and reliability of the applied multi-scale analysis by comparing the numerical results with those derived experimentally. The essential findings are finally summarized in Section 5.

\section{Image analysis and construction of the geometrical model}

It has been demonstrated in our previous work, see e.g. [9, 1, 17, 18] that image analysis of real, rather then artificial, material systems plays an essential role in the derivation of a reliable and accurate computational model. This issue is revisited here for the case of woven fabric $\mathrm{C} / \mathrm{C}$ laminate with particular relation to the adopted uncoupled multi-scale solution strategy.

Table 1: Material parameters of individual phases [19, 20]

\begin{tabular}{|c|c|c|c|}
\hline Material & $\begin{array}{c}\text { Thermal conductivity } \\
{\left[\mathrm{Wm}^{-1} \mathrm{~K}^{-1}\right]}\end{array}$ & $\begin{array}{c}\text { Specific heat } \\
{\left[\mathrm{Jkg}^{-1} \mathrm{~K}^{-1}\right]}\end{array}$ & $\begin{array}{c}\text { Mass density } \\
{\left[\mathrm{kgm}^{-3}\right]}\end{array}$ \\
\hline Carbon fibers & $(0.35,0.35,35)$ & 753 & 1810 \\
Carbon matrix & 6.3 & 1256 & 1400 \\
Voids filled with air & 0.02 & 1000 & 1.3 \\
\hline
\end{tabular}

For illustration, let us now consider an eight-layer carbon-carbon composite laminate. Individual plies are made of plain weave carbon fabric Hexcel 1/1 embedded in a carbon matrix. Each filament (fiber tow) contains about 6000 carbon fibers T800H based on Polyacrylonitril precursor. Such fibers are known as having a relatively low orderliness of graphen planes on nano-scale. Nevertheless, unlike glassy carbon, they still posses a transverse isotropy with the value of longitudinal thermal conductivity considerably exceeding the one in the transverse direction, see Table 1. As already mentioned in the introductory part the carbon matrix forms as a result of several cycles of carbonization/densification and final graphitization of the initial polymeric precursor (green composite) where the phenolic resin UMAFORM LE is used as the bonding agent (matrix). Note that phenolic resins belong to a family of non-graphitizing resins so that the final carbon matrix essentially complies, at least in terms of its structure, with the 


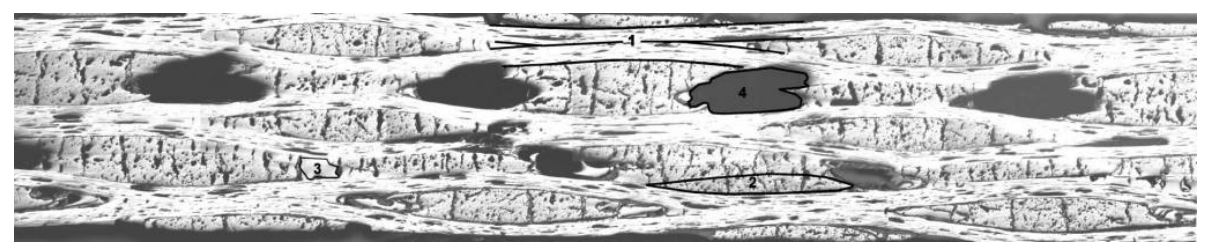

Figure 2: Representative segment of eight-layer plain weave fabric laminate.

original cross-linked polymeric precursor. Therefore, the resulting material symmetry is more or less isotropic with material parameters corresponding those of glassy carbon [21, 22].

A typical segment of the composite laminate appears in Fig. 2 showing characteristic porosity which may exceed at the structural level (macroscale) 30\% [23, 24] and is often considered as an intrinsic property of this type of composite [21]. Several such micrographs were processed with the help of LUCIA G [25], Adobe Photoshop, Corel Draw and Matlab R12 softwares to acquire information regarding the basic structural units like an average thickness of carbon tows, size of voids, shape and essential dimensions of fiber tow cross-section, distribution of transverse and delamination cracks etc. which were subsequently exploited in the construction of representative unit cells on individual scales.

\subsection{Micro-scale}

Starting with the fiber tow composite as the basic structural element we recall Fig. 1 showing a typical shape of the fiber tow cross-section and significant amount of transverse cracks and voids resulting in a non-negligible porosity up to $15 \%$. Unfortunately, a detailed analysis of the fiber tow cross-section would be computationally infeasible. As a suitable method of attack appears on the other hand formulation of a two-step homogenization problem.

To proceed, let us consider a typical micrograph of the fiber matrix composite shown in Fig 3(b) taken as a random cut from the fiber tow cross-section evident in Fig. 3(a). Providing this section is sufficiently large to be statistically representative of a real microstructure it becomes possible to proceed in the footsteps of our previous work [9] and formulate a statistically equivalent periodic unit cell at the level of individual fibers. Keeping in mind on the other hand a relatively high volume fraction of fibers, approx. 55\%, the results presented in [9] and remarks put forward in [26] allows us to conclude that the actual microstructure can be replaced with a simple periodic hexagonal unit cell plotted in Fig. 3(d). The resulting effective material parameters then serve as direct input for the analysis at the level of the fiber tow cross-section. Here, a new unit cell, again exploiting information acquired from image analysis, is introduced to properly account for the porous phase. The proposed periodic unit cell, which not only reflects the voids volume fraction but to some extent also their arrangement, is seen in Fig. 3(c).

\subsection{Meso-scale}

Having derived the effective material parameters for the fiber tow composite, the assumed three level homogenization procedure continues along the same lines on meso-scale. To that end, let us recall the representative section of the composite laminate in Fig. 2. A detailed inspection of this micrograph reveals three more or less periodically repeated geometries. For better view we refer to Figs. 4(a)-(c).

Several such sections taken from various locations of the laminated plates were examined, again with the help of image analyzer LUCIA G, to obtain averages of various parameters including segment dimensions, fiber tow thickness, shape of the fiber tow cross-section also 


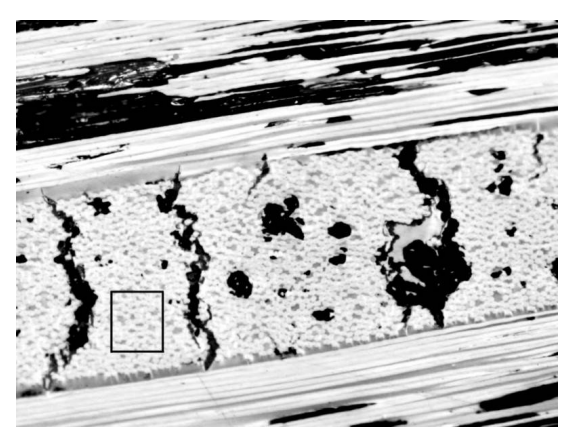

(a)

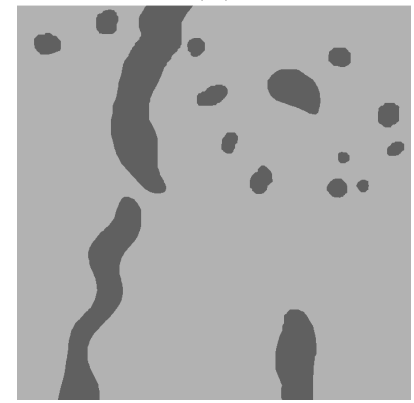

(c)

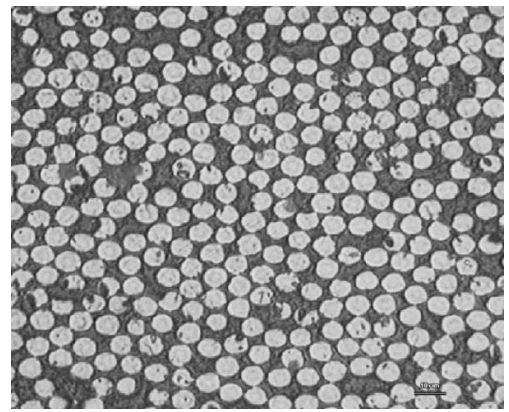

(b)

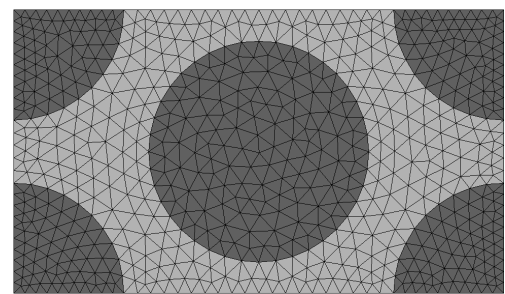

(d)

Figure 3: Homogenization on micro-scale: (a) fiber tow composite, (b) fiber-matrix composite, (c) voids-composite periodic unit cell, (d) fiber-matrix periodic unit cell

position, size and location of large vacuoles. Approximately 100 measurements were carried out for each segment and subsequently utilized in the formulation of corresponding periodic unit cells displayed in Figs. 4(d)-(f).

\subsection{Macro-scale}

The final, clearly the most simple, step requires a construction of the homogeneous, thought not isotropic, laminated plate. The stacking sequence of individual periodic unit cells, which were introduced in the previous section, complies with that observed for the actual composite sample. Partially for the sake of simplicity, but also to be consistent with the analyses performed on lower scales, the periodic boundary conditions are considered even on the macro-scale. Providing we are interested only in the bulk response of the laminate thus ignoring detailed variation of the local fields, this assumption does not yield a significant error in the desired estimates of the macroscopic coefficients of thermal conductivities. Clear evidence is available in Section 4 comparing the numerical and experimental results where the former ones are derived from the theoretical grounds presented in the next section.

\section{Theoretical formulation}

We now proceed to establish a framework for the determination of the effective thermal conductivities regardless of the material system considered in the previous section providing the same boundary conditions are applied on each scale. An attentive reader will notice a close similarity with the derivation of the effective elastic material constants, see e.g. [11] for that matter. 
(a)

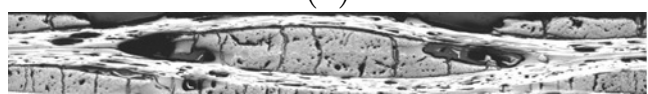

(b)

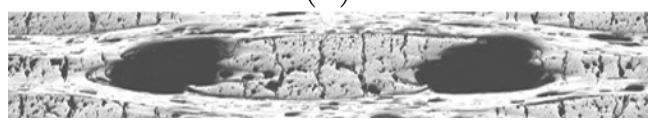

(c) (d)

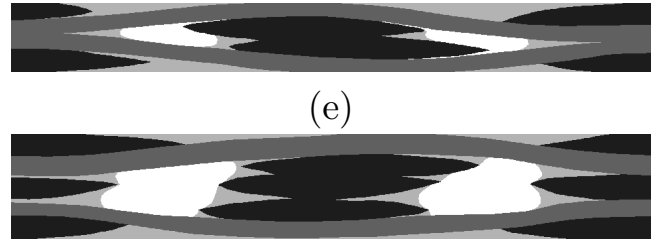

(f)

Figure 4: Homogenization on meso-scale: (a)-(b) PUC1 representing carbon tow-carbon matrix composite, (c)-(d) PUC2 with vacuoles aligned with delamination cracks due to slip of textile plies, (e)-(f) PUC3 with extensive vacuoles representing the parts with textile reinforcement reduction due to bridging effect in the middle ply

\subsection{Governing equations}

Adhering to indicial notation with $\dot{a}=\frac{\partial a}{\partial t}$ and $a_{, i}=\frac{\partial a}{\partial x_{i}}$ representing the time and space derivatives, respectively, the simplest form of the balance equation reads

$$
\left(\rho^{s} C_{p}^{s}\right) \dot{\theta}+q_{i, i}^{s}=0
$$

where $\rho^{s}$ is the mass density and $C_{p}^{s}$ represents the specific heat of a given phase $s$. If referring to micro-scale, for example, the superscript $s$ may first represent the fiber or the matrix phase and in the second step of homogenization, recall Section 2.1, it may be associated with the fiber-matrix composite and voids. The phase constitutive equations follow from the generalized version of Fourier's law and are provided by

$$
q_{i}^{s}=-\chi_{i j}^{s} h_{i}^{s}, \quad h_{i}^{s}=-\psi_{i j}^{s} q_{i}^{s}, \quad \text { in } \Omega^{s},
$$

where $h_{i}=\theta_{, i}, \boldsymbol{\chi}$ is the thermal conductivity matrix $\left[\mathrm{Wm}^{-1} \mathrm{~K}^{-1}\right]$ and $\boldsymbol{\psi}=\chi^{-1}$ is the thermal resistivity matrix. Providing a heat flux, consistent with Eq. $(2)_{2}$, is imposed over the entire boundary $\Gamma$ we arrive, with the help of Eq. $(4)_{1}$, at the following boundary condition

$$
\bar{q}_{n}=-n_{i} \chi_{i j} \theta_{, j} \quad \text { on } \Gamma .
$$

The weak form of Eqs. (3)- (5) is then given by

$$
\int_{\Omega}\left[\delta \theta\left(\rho C^{p}\right) \dot{\theta}+\delta \theta_{, i} \chi_{i j} \theta_{, j}\right] \mathrm{d} \Omega+\int_{\Gamma} \delta \theta \bar{q}_{n} \mathrm{~d} \Gamma=0 .
$$

It is interesting to show that under the steady-state conditions $(\dot{\theta}=0)$. Eq. (6) is essentially equivalent to

$$
\left\langle\delta \theta_{, i} \chi_{i j} \theta_{, j}\right\rangle=-\delta H_{i} Q_{i},
$$

where $\langle a\rangle$ represents a volume average of a given quantity, i.e. $\langle a\rangle=\frac{1}{|\Omega|} \int_{\Omega} a \mathrm{~d} \Omega$. This becomes evident after introducing Eqs. (1) and (2) 2 into the second term of Eq. (6) to get

$$
\begin{aligned}
\frac{1}{|\Omega|} \int_{\Gamma} \delta\left(H_{i} x_{i}+\theta^{*}\right) Q_{j} n_{j} \mathrm{~d} \Gamma & =\frac{1}{|\Omega|}\left[\delta H_{i} Q_{j} \int_{\Gamma} x_{i} n_{j} \mathrm{~d} \Gamma+\int_{\Gamma} \delta \theta^{*} Q_{j} n_{j} \mathrm{~d} \Gamma\right] \\
& =\delta H_{i} Q_{j} \delta_{i j}=\delta H_{i} Q_{i} .
\end{aligned}
$$


The integral $\int_{\Gamma} \delta \theta^{*} Q_{j} n_{j} \mathrm{~d} \Gamma$ disappears providing we set the fluctuation part of the temperature field $\theta^{*}$ equal to zero on $\Gamma$ or impose the periodic boundary conditions (the same values of $\theta^{*}$ on opposite sides of a rectangular periodic unit cell). Either choice of variation of $\theta^{*}$ on $\Gamma$ readily ensures that $\left\langle h_{i}\right\rangle=H_{i}$ since

$$
H_{i}=\frac{1}{|\Omega|} \int_{\Omega} h_{i} \mathrm{~d} \Omega=H_{i}+\frac{1}{|\Omega|} \underbrace{\int_{\Gamma} \theta^{*} n_{i} \mathrm{~d} \Gamma}_{=0},
$$

where integration by parts was used to transform the volume integral into the integral over the boundary $\Gamma$. As already mentioned in the introductory part, the periodic boundary conditions will be used in the actual numerical analysis.

Note that Eq. (7) essentially resembles the Hill lemma in the context of pure thermomechanical problem [12]. Here it shows the consistency of the entropy change at the two associated scales (micro-meso, meso-macro) [27, 10].

The boundary conditions $(2)_{2}$ further imply the volume average of the local heat flux be equal to the prescribed macroscopic heat flux $Q_{i}$

$$
\left\langle q_{i}\right\rangle=Q_{i} .
$$

This immediately follows under steady state conditions since $q_{i, i}=0$ so that $q_{i}=\left(q_{j} x_{i}\right)_{, j}$. The volume average of the local quantity $q_{i}$ then gives, see also [10],

$$
\begin{aligned}
|\Omega|\left\langle q_{i}\right\rangle & =\int_{\Omega} q_{i} \mathrm{~d} \Omega=\int_{\Omega}\left(q_{j} x_{i}\right)_{, j} \mathrm{~d} \Omega \\
& =\int_{\Gamma} q_{j} n_{j} x_{i} \mathrm{~d} \Gamma=\int_{\Gamma} q_{n} x_{i} \mathrm{~d} \Gamma=\int_{\Gamma} Q_{j} n_{j} x_{i} \mathrm{~d} \Gamma \\
& =Q_{j} \int_{\Omega} x_{i, j} \mathrm{~d} \Omega=|\Omega| Q_{j} \delta_{i j}=|\Omega| Q_{i} .
\end{aligned}
$$

\subsection{Effective conductivity and resistivity matrices}

To proceed, we limit our attention to steady state conditions and employ, in view of the forthcoming finite element formulation, the standard matrix notation; e.g. [28]. Then, under pure thermal loading consistent with the boundary conditions $(2)_{1}$ and taking into account the fact that variation of a prescribed quantity vanishes, we receive the following form of Eq. (7)

$$
\left\langle\delta\{\mathrm{h}\}^{\top}[\chi]\{\mathrm{h}\}\right\rangle=0,
$$

In the framework of the finite element method (FEM) the vector $\{h\}$, recall Eq. (1), is provided by

$$
\{\mathrm{h}\}=\{\mathrm{H}\}+[\mathrm{B}]\left\{\theta_{\mathrm{d}}^{*}\right\},
$$

where $[B]$ stores the derivatives of the shape functions and $\left\{\theta_{d}^{*}\right\}$ lists the nodal values of the fluctuation part of the temperature field. Substituting from Eq. (13) back into Eq. (12) yields the resulting system of equations

$$
[\mathrm{K}]\left\{\theta_{\mathrm{d}}^{*}\right\}=\{\mathrm{R}\},
$$

where

$$
\begin{aligned}
{[\mathrm{K}] } & =\int_{\Omega}[\mathrm{B}]^{\top}[\chi][\mathrm{B}] \mathrm{d} \Omega, \\
\{\mathrm{R}\} & =-\int_{\Omega}[\mathrm{B}]^{\top}[\chi]\{\mathrm{H}\} \mathrm{d} \Omega=-[\mathrm{S}]^{\top}\{\mathrm{H}\} .
\end{aligned}
$$


Solving for $\left\{\theta_{\mathrm{d}}^{*}\right\}$ from Eq. (14) gives

$$
\left\{\theta_{\mathrm{d}}^{*}\right\}=-[\mathrm{K}]^{-1}[\mathrm{~S}]^{\top}\{\mathrm{H}\}=-[\mathrm{G}]\{\mathrm{H}\} .
$$

Next, introducing the vector $\left\{\theta_{\mathrm{d}}^{*}\right\}$ back into Eq. (13) provides the volume average of the local heat flux in the form

$$
\{\mathrm{Q}\}=\langle\{\mathrm{q}\}\rangle=-\frac{1}{|\Omega|} \int_{\Omega}[\chi][[\mathrm{I}]-[\mathrm{B}][\mathrm{G}]] \mathrm{d} \Omega\{\mathrm{H}\} .
$$

Finally, writing the macroscopic constitutive law in the form

$$
\{\mathrm{Q}\}=-[\chi]^{\text {hom }}\{\mathrm{H}\},
$$

readily provides the homogenized effective conductivity matrix $[\chi]^{\text {hom }}$ as

$$
[\chi]^{h o m}=\frac{1}{|\Omega|} \int_{\Omega}[\chi][[I]-[B][G]] \mathrm{d} \Omega .
$$

In actual computations the coefficients of the effective conductivity matrix $[\chi]^{\text {hom }}$ are found as volume averages of the local fields from the solution of three successive steady state heat conduction problems. To that end, the periodic unit cell is loaded, in turn, by each of the two (2D) or three (3D) components of $\{\mathrm{H}\}$, while the others vanish. The volume flux averages normalized with respect to $\{\mathrm{H}\}$ then furnish individual columns of $[\chi]^{\text {hom }}$. The required periodicity conditions (the same temperatures $\left\{\theta_{d}^{*}\right\}$ on opposite sides of the unit cell) are accounted for through multi-point constraints. In our particular case it suffice to assign the same code numbers to respective periodic pairs.

The derivation of the effective resistivity matrix may proceed along the same lines providing the unit cell is loaded by the prescribed macroscopic uniform heat flux $\{Q\}$. In this particular case, the volume average of the local temperature gradient is not known a priori. Eq. (7) then yields two sets of governing equations for unknown nodal values of $\theta^{*}$ and volume average of the local temperature gradient $\langle\{\mathrm{h}\}\rangle=\{\mathrm{H}\}$ in the form

$$
\left[\begin{array}{cc}
{[\mathrm{L}]} & {[\mathrm{S}]} \\
{[\mathrm{S}]^{\mathrm{T}}} & {[\mathrm{K}]}
\end{array}\right]\left\{\begin{array}{c}
\{\mathrm{H}\} \\
\left\{\theta_{\mathrm{d}}^{*}\right\}
\end{array}\right\}=\left\{\begin{array}{c}
-\{\mathrm{Q}\} \\
\{0\}
\end{array}\right\}
$$

where the matrices $[\mathrm{S}]$ and $[\mathrm{K}]$ were already introduced by Eqs. (15) and (16) and the matrix [L] is given by

$$
[\mathrm{L}]=\int_{\Omega}[\chi] \mathrm{d} \Omega .
$$

Combining the above equation together with the macroscopic constitutive law

$$
\{\mathrm{H}\}=-[\psi]^{\text {hom }}\{\mathrm{Q}\},
$$

gives after some manipulations the searched homogenized effective resistivity matrix $[\psi]^{\text {hom }}$ in the form

$$
[\psi]^{h o m}=\left[[\mathrm{L}]-[\mathrm{S}][\mathrm{K}]^{-1}[\mathrm{~S}]^{\top}\right]^{-1} .
$$




\section{Results of the numerical analysis}

The present section summarizes the numerical results of the proposed three step uncoupled homogenization scheme. On each scale the relevant periodic unit cell developed in Section 2 was discretized into finite elements. The geometrical complexity of individual computational models together with the desired periodicity constraints led to extremely fine meshes. While this may seem irrelevant from the steady state conditions point of view, it suddenly becomes very important when running the transient heat conduction problem. This is also why the periodic boundary conditions were disregarded in the latter case. While the results are again presented separately for individual scales, it is worthwhile mentioning that the output in terms of the effective conductivities derived at a lower scale served directly as the input for the up-scale analysis. Regardless of the type of analysis performed, steady state or transient conduction problem, only the boundary conditions of type $(2)_{1}$ were considered and the effective conductivities were found through the procedure described in Section 3.2.

\subsection{Micro-scale}

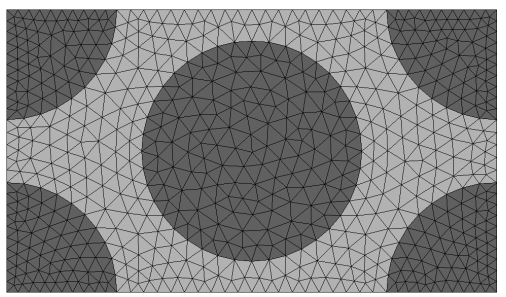

(a)

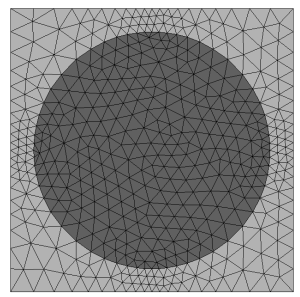

(b)

Figure 5: Finite element mesh of fiber tow: (a) hexagonal arrangement of fibers, (b) square arrangement of fibers

As suggested in Section 2.1 the effective conductivities of the fiber tow composite were derived in two steps. First, an intact carbon fiber-carbon matrix composite was considered. Two computational models displayed in Fig. 5 were examined. Both 2D and 3D analysis was carried out. A simple rule of mixture (RM) was used to estimate the effective thermal conductivities in the fiber direction ( $z$-direction) for comparison with the general 3D analysis

$$
k_{z}=c^{f} k_{z}^{f}+c^{m} k_{z}^{m},
$$

where $c^{f}, c^{m}, k_{z}^{f}, k_{z}^{m}$ represent the fiber and matrix volume fractions and conductivities in the $z$-direction, respectively. Their values are listed in Table 1 . The homogenized conductivities then appear in Table 2.

Table 2: Effective thermal conductivities $\left[\mathrm{Wm}^{-1} \mathrm{~K}^{-1}\right]$ - Intact fiber tow

\begin{tabular}{|c|c|c|c|c|}
\hline Geometry & $k_{x}$ & $k_{y}$ & $k_{z}-3 \mathrm{DFEM}$ & $k_{z}-\mathrm{RM}$ \\
\hline hexagonal & 2.17 & 2.17 & 21.93 & 21.96 \\
square & 2.10 & 2.10 & 22.04 & 21.96 \\
\hline
\end{tabular}

As expected, there is a minor difference in the results provided by both models. Nevertheless, the effective values derived from the hexagonal array model were further employed in 
the subsequent analysis step which allowed us to introduce the intra-tow voids into the intact but already homogeneous fiber tow composite. Again, two computational models evident from Fig. 6 were studied.

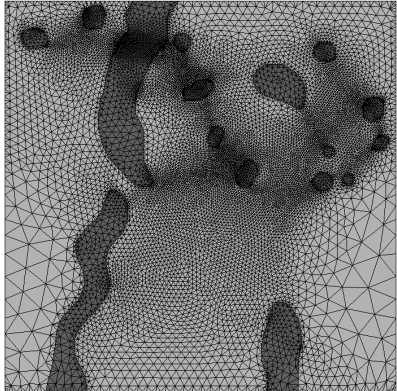

(a)

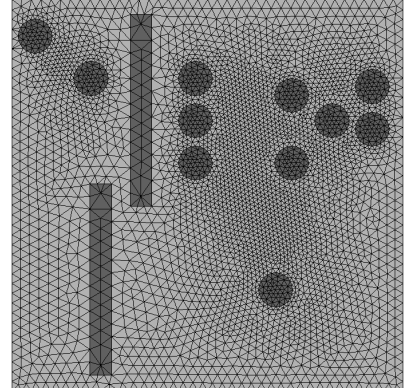

(b)

Figure 6: Finite element mesh of fiber tow composite including voids: (a) real distribution of voids, (b) approximate distribution of voids

Hereafter, we were concerned with the two-dimensional problem only. The corresponding results are available in Table 3. Apart from a steady state analysis a transient heat conduction problem goverened by Eq. (3) was addressed. Clearly, solving this problem then calls for the effective mass density and specific heat on the level of an intact fiber/matrix composite. For this purpose a logarithmic rule of mixture was adopted in the form

$$
\begin{aligned}
\ln \rho & =c^{f} \ln \rho^{f}+c^{m} \ln \rho^{m}, \\
\ln C_{p} & =c^{f} \ln C_{p}^{f}+c^{m} \ln C_{p}^{m} .
\end{aligned}
$$

The analysis was performed with the help of FEMlab commercial code using the Heat Transfer Mode [29]. To simulate a unidirectional flow the unit cell was heated on the one side while zero temperature was prescribed on the other side. The initial temperature was also assumed to be equal to zero. In addition, a zero flux boundary conditions were prescribed on the remaining sides to represent isolated surfaces. This clearly yields the mixed boundary conditions on $\Gamma$, which in turn may violate the conditions (9) and (11) if the periodic boundary conditions are disregarded, see the discussion in the following paragraphs. The conservation condition (7), however, holds even in this case.

The thermal conductivities were then estimated from Eq. (19) after reaching the steady state conditions (time independent temperature profile). In such a case Eq. 3 reduces to $q_{i, i}=0$ thus naturally enforcing Eq. (11). If for example a unidirectional heat flow along the macroscopic $x$ axis is considered, then the component of the effective heat conductivity is, in view of Eq. (19), provided by

$$
\chi_{x}^{h o m}=-\frac{\left\langle q_{x}\right\rangle}{\left\langle\theta_{, x}\right\rangle} .
$$

The $\chi_{y}{ }^{\text {hom }}$ component of the effective conductivity matrix $[\chi]^{\text {hom }}$ is derived analogously. If no action is taken this result corresponds to the assumption of $t^{*}=0$ on $\Gamma$.

The results in Table 3 suggest a minor difference between the steady state and transient heat analysis. Among others the lack of periodic boundary conditions, much coarser mesh [6] and truly approximate estimates of the homogenized mass density and specific heat appear as the most critical sources for the resulting difference. One may also expect this difference to grow when moving up the scales particularly if assuming a certain consistency of the three step 
Table 3: Effective thermal conductivities $\left[\mathrm{Wm}^{-1} \mathrm{~K}^{-1}\right]$ - Fiber tow with hexagonal arrangement of fibers including voids

\begin{tabular}{|c|c|c|c|c|c|c|}
\hline Analysis & \multicolumn{3}{|c|}{ Steady state } & \multicolumn{3}{c|}{ Transient } \\
\hline Voids distribution & $k_{x}$ & $k_{y}$ & $k_{z}-\mathrm{RM}$ & $k_{x}$ & $k_{y}$ & $k_{z}-\mathrm{RM}$ \\
\hline real & 0.97 & 1.70 & 19.01 & 1.20 & 1.60 & 19.10 \\
approximate & 1.12 & 1.77 & 19.01 & - & - & - \\
\hline
\end{tabular}

procedure in the sense that the results derived on a lower scale using one type of analysis are transferred to a higher scale where the same type of analysis is performed.

\subsection{Meso-scale}

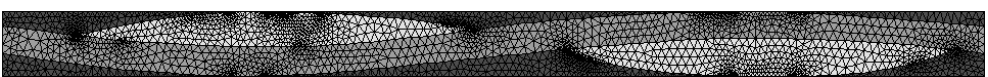

(a)

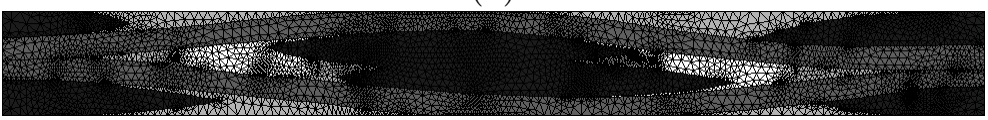

(b)

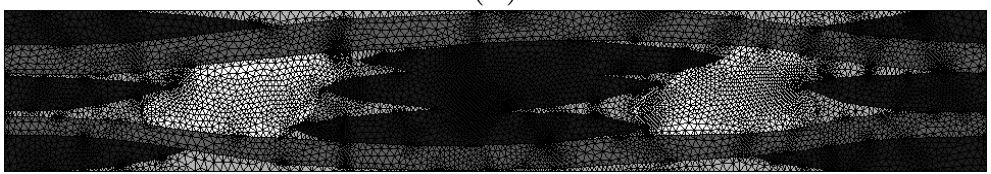

(c)

Figure 7: Finite element meshes; (a) PUC1, (b) PUC2, (c) PUC3.

At this level the carbon fiber tow is treated as a homogeneous phase with the effective material parameters derived from the numerical analysis on micro-scale. Since these are provide in the local (fiber) coordinate system, it is desirable, in order to account for the tow waviness, to transform the corresponding effective conductivity matrix into the global coordinate system as

$$
[\chi(\boldsymbol{x})]_{g}^{\text {tow }}=[\mathbf{T}(\boldsymbol{x})]^{\mathbf{T}}[\chi]_{l}^{\text {tow }}(\mathbf{x})[\mathbf{T}(\boldsymbol{x})],
$$

where $[\mathbf{T}(\boldsymbol{x})]$ is the continuously varying transformation matrix. The matrix $[\chi(\boldsymbol{x})]_{g}^{\text {tow }}$ then enters Eq. (20) where applicable. The computational procedure is, nevertheless, identical to the one outlined in the previous section. With reference to Section 2.2 the three periodic unit cells in Fig. 7 were examined. Table 4 then provides the resulting homogenized thermal conductivities. To accept the notable difference in the results from the two types of distinct analyses we address the reader to the comments offered in the last paragraph of Section 4.1.

Although more complicated, solving the transient heat conduction problem allows us to receive further information regarding the response of a composite to thermal loading including a gradual evolution of the temperature profile and time lag to reach the steady state conditions. Fig. 8 shows a typical graphical output of the results provided by FEMlab. These plots, in particular, correspond to the onset of steady state conditions showing already more or less constant temperature gradient (linear variation of temperature) for all unit cells with corresponding distribution of heat fluxes. 

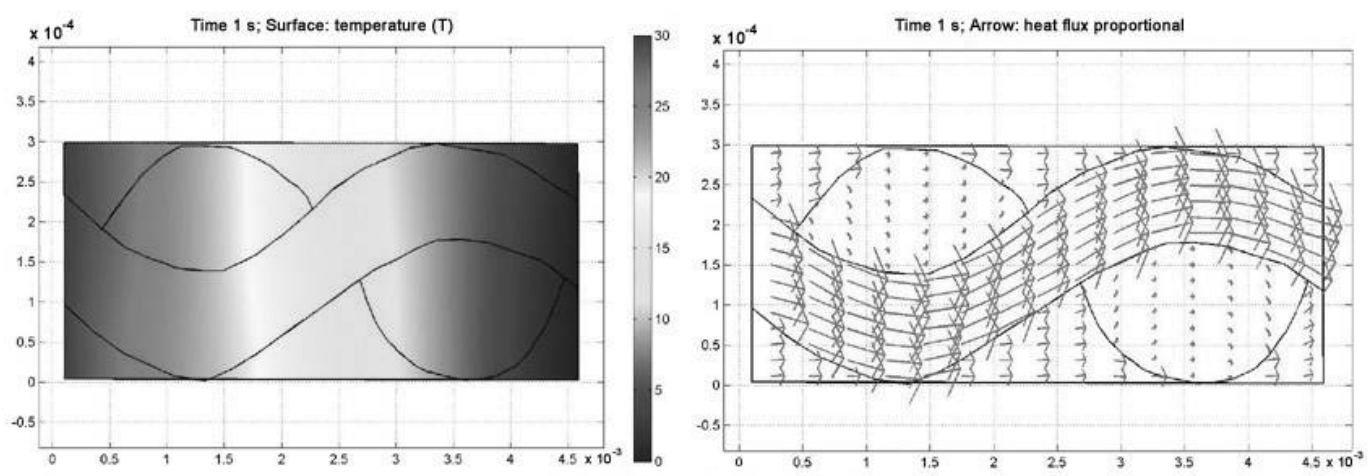

(a)
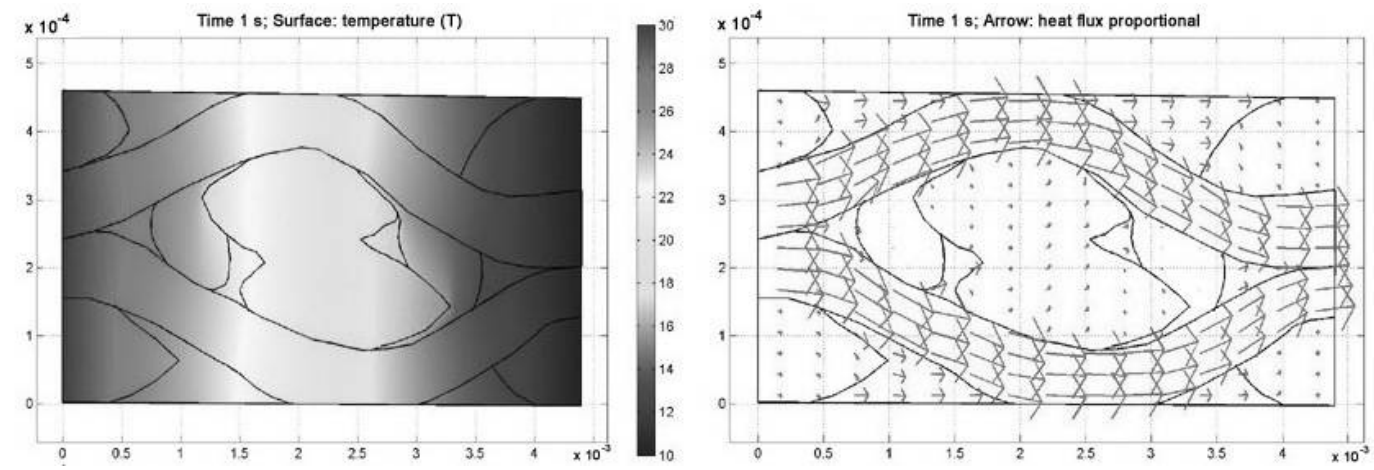

(b)
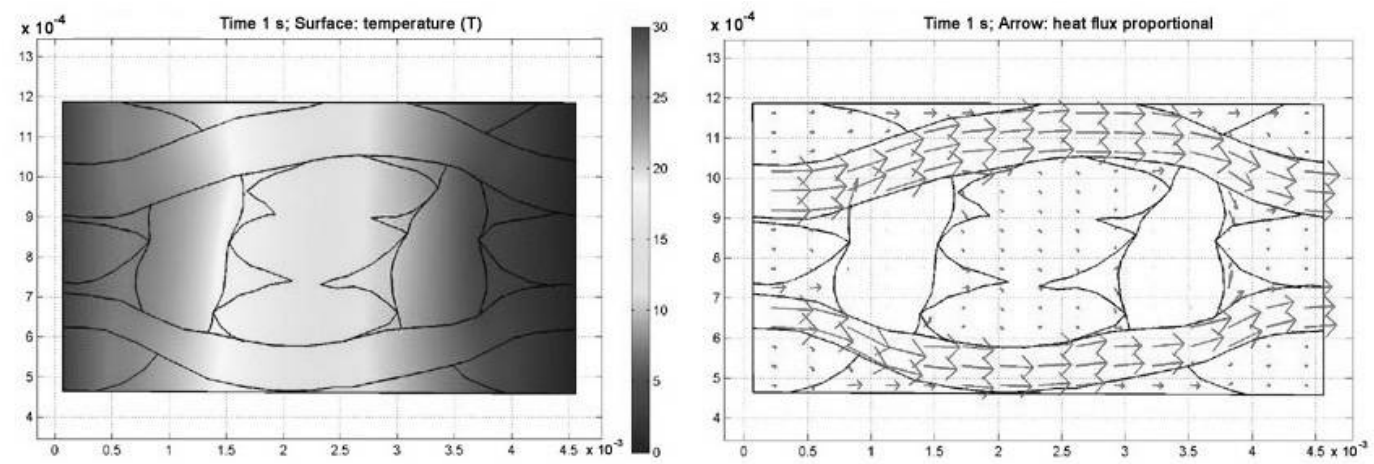

(c)

Figure 8: Simulation of transient heat conduction problem - temperature profile and heat flux in the direction parallel to the composite plate: (a) PUC1, (b) PUC2, (c) PUC3 
Table 4: Effective thermal conductivities $\left[\mathrm{Wm}^{-1} \mathrm{~K}^{-1}\right]$ - Representative unit cells of textile composites

\begin{tabular}{|c|c|c|c|c|}
\hline Analysis & \multicolumn{2}{|c|}{ Steady state } & \multicolumn{2}{c|}{ Transient } \\
\hline Cell & $k$-longitudinal & $k$-transverse & $k$-longitudinal & $k$-transverse \\
\hline PUC 1 & 9.46 & 2.27 & 9.10 & 2.30 \\
PUC 2 & 9.03 & 1.47 & 7.40 & 1.80 \\
PUC 3 & 7.29 & 1.53 & 6.60 & 1.75 \\
\hline
\end{tabular}

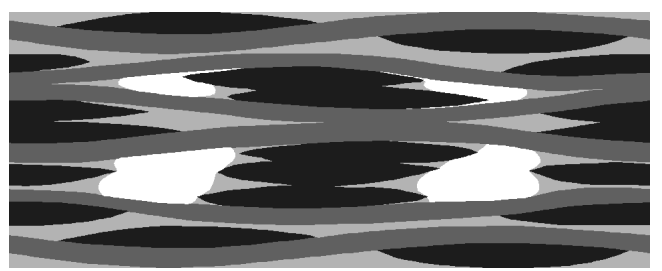

(a)

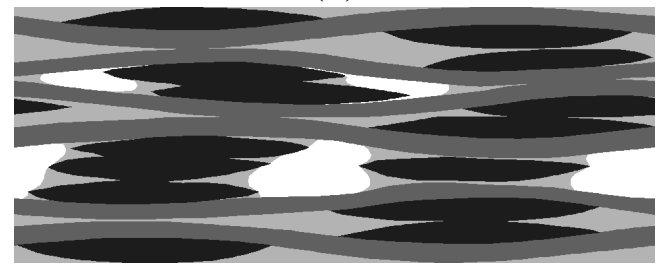

(c)

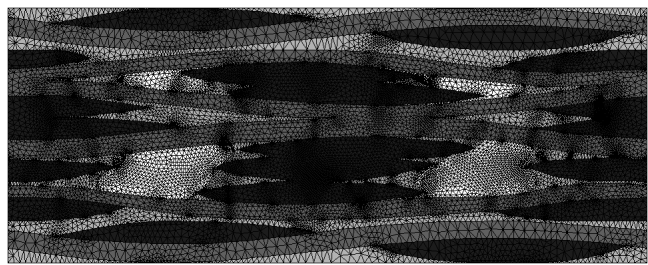

(b)

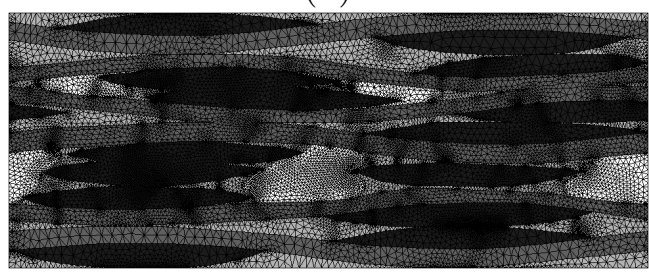

(d)

Figure 9: Textile laminate with: (a)-(b) regular arrangement of plies, (c)-(d) irregular arrangement of plies.

\subsection{Macro-scale}

The macroscopic analysis of heat conduction problem represents the final step of the proposed multiscale approach. From the computational point of view it requires a relatively simple analysis of a three-layer laminate stacked from three periodic unit cells shown Fig. 7. However, the geometrical details of these unit cells are no longer relevant. Instead, they are treated as homogeneous blocks with the assigned homogenized (mesoscopic) properties. The resulting macroscopic properties are stored in Table 5.

To validate these results an additional numerical analysis was performed. Here, all geometrical details of the laminated plate were taken into account by creating a multi-layered periodic unit cell exploiting the geometrical models developed for the mesoscopic analysis. The selected stacking sequence was motivated by images of the real laminate such as the one displayed in Fig. 2. Two such unit cells were constructed, see Fig. 9. While the first unit cell, Fig. 9(a), assumed an ideal stacking with regular arrangement of individual unit cells, the second one, Fig. 9(c), allowed for a mutual shift of individual plies to approximate the actual geometry more accurately. It is interesting to see, Table 5, that all types of analyses (laminate, PUC with regular and PUC with irregular arrangement of unit cells) essentially provide the same estimates of the macroscopic thermal conductivities. Such a conclusion clearly advocates the use of multiscale analysis at least in the present context of linear steady state or transient heat conduction problem.

To judge the quality of any computational approach purely from numerical experiments seems, however, rather shallow. To enhanced credit of a numerical analysis it is therefore 
Table 5: Effective thermal conductivities $\left[\mathrm{Wm}^{-1} \mathrm{~K}^{-1}\right]$ - Laminated plate (The number in parentheses indicates the difference between a numerical value and experimental data.)

\begin{tabular}{|c|c|c|c|c|}
\hline Analysis & \multicolumn{2}{|c|}{ Steady state } & \multicolumn{2}{c|}{ Transient } \\
\hline Geometry & $k$-longitudinal & $k$-transverse & $k$-longitudinal & $k$-transverse \\
\hline Laminate & $8.47(15.3 \%)$ & $1.66(3.75 \%)$ & $8.30(17.0 \%)$ & $1.80(12.5 \%)$ \\
Macro cell regular & $8.65(13.5 \%)$ & $1.68(5.00 \%)$ & - & - \\
Macro cell irregular & $8.60(14.0 \%)$ & $1.67(4.37 \%)$ & - & - \\
Measured & - & - & 10.00 & 1.60 \\
\hline
\end{tabular}

desirable to compare the numerical results with those obtained experimentally. For this type of composite the results from experimental investigation of the thermophysical properties are available in [30]. For details on the pulse transient method used in this work together with computational models required to relate temperature to the generated heat pulse we refer the reader to the above paper. Some basic information can also be found in [6]. The experimentally determined macroscopic conductivities appear in Table 5. Taking into account the possible errors in the determination of phase material parameters (carbon fibers and carbon matrix) on the one hand and errors associated with the laboratory measurements on the other hand adds further confidence in the presented three-level uncoupled multiscale homogenization approach. In this context, the simplified two-dimensional approach (with the exception to the lowest scale) adopted for the solution of a generally three-dimensional problem, a natural component of laboratory measurements, should also be added to a list of sources of possible errors. It is worth noting even under these simplifications, the errors associated with the analysis compare well with the results of fully 3D analyses reported in [4]. Therefore, it is expected that a reliable three-dimensional geometrical model constructed on the mesoscopic level will further improve the predictive capabilities of the multiscale solution strategy. This is the topic of our current research.

\section{Conclusions}

Three levels of hierarchy are introduced in this contribution to derive the effective thermal conductivities of a plain weave textile laminate. Different resolution of microstructural details are considered on individual scales for the construction of an adequate representative unit cell. Such a unit cell arises as a result of elaborate evaluation of images of a real composite sample. The geometrical complexity of these types of composites are mainly responsible for a slow progress in the formulation of a generally three-dimensional unit cell. Possibility to enlighten this subject is given in [1] promoting the construction of such an RVE by matching statistical characteristics of both the real composite and RVE, at this step, however, in the absence of a porous phase. Owing to a significant contribution of this phase to the overall volume of the composite ruled the choice of a simplified two-dimensional analysis for the present study.

Clearly, the theoretical formulation, here developed on the basis of the first-order homogenization, is quite general and space invariant. Both transient and steady state conditions were examined in this study. When considering solely the temperature boundary conditions of type $(2)_{1}$ allows the solution to be accepted even if neglecting the periodic boundary conditions. Proper averaging relations then still provide the correct means for the scale transition. This has been exploited when running the transient heat conduction problem. The periodic constraints, on the other hand, were imposed for the estimates of effective thermal conductivities under the steady state conditions. In this case, too fine discretization needed for the straightforward 
introduction of periodic boundary conditions was of minor concern. Both approaches have shown, however, their potential in the derivation of the desired effective (homogenized) thermal conductivities of highly complex plain weave textile composites through the application of fully uncoupled multiscale homogenization scheme.

\section{Acknowledgments}

The financial support provided by the GAČR grant No. 106/07/1244 and partially also by the research project CEZ MSM 6840770003, is gratefully acknowledged.

\section{References}

[1] J. Zeman and M. Šejnoha. Homogenization of balanced plain weave composites with imperfect microstructure: Part i - theoretical formulation. International Journal for Solids and Structures, 41(22-23):6549-6571, 2004.

[2] M. Palán. Thermal properties of $C$ - $C$ composites reinforced by textile fabric. PhD thesis, TU Liberec, 2002. in Czech.

[3] A. Dasgupta, R. K. Agarwal, and S. M. Bhandarkar. Three-dimensional modeling of woven-fabric composites for effective thermo-mechanical and thermal properties. Composites Science and Technology, 56(3):209-223, 1996.

[4] K. Woo and N.S. Goo. Thermal conductivity of carbon-phenolic 8-harness satin weave composites. Composite Structures, 66(1-4):521-526, 2004.

[5] P. Del Puglia, M. A. Sheikh, and D. R. Hayhurst. Modelling the degradation of thermal transport in a cmc material due to three different classes of porosity. Modelling and Simulation in Materials Science and Engineering, 12(2):357-372, 2004.

[6] B. Tomková. Modelling of thermophysical properties of woven composites. $\mathrm{PhD}$ thesis, TU Liberec, 2006.

[7] Y. Benveniste, T. Chen, and G.J. Dvorak. The effective thermal conductivity of composites reinforced by coated cyllindrically orthotropic fibers. Journal of Applied Physics, 67(6):2878-2884, 1990.

[8] J. Zeman and M. Šejnoha. From random microstructures to representative volume elements. Modelling and Simulation in Materials Science and Engineering, 15(4):S325-S335, 2007.

[9] J. Zeman and M. Šejnoha. Numerical evaluation of effective properties of graphite fiber tow impregnated by polymer matrix. Journal of the Mechanics and Physics of Solids, 49(1):69-90, 2001.

[10] I. Özdemir, W. A. M. Brekelmans, and M. G. D. Geers. Computational homogenization for heat conduction in heterogeneous solids. International Journal for Numerical Methods in Engineering, 73(2):185-204, 2008.

[11] J. C. Michel, H. Moulinec, and P. Suquet. Effective properties of composite materials with periodic microstructure: A computational approach. Computer Methods in Applied Mechanics and Engineering, 172:109-143, 1999. 
[12] M. Šejnoha and J. Zeman. Micromechanical analysis of random composites, volume 6 of CTU Reports. Czech Technical University in Prague, 2002. http://mech.fsv.cvut.cz/ $\sim$ sejnom/download/hab.pdf.

[13] O. van der Sluis, P. J. G. Schreurs, W. A. M Brekelmans, and H. E. H. Meijer. Overall behaviour of heterogeneous elastoviscoplastic materials: effect of microstructure modelling. Mechanics of Materials, 32:449-462, 2000.

[14] K. Terada, M. Hori, T. Kyoya, and N. Kikuchi. Simulation of the multi-scale convergence in computational homogenization approaches. International Journal of Solids and Structures, $37(16): 2285-2311,2000$.

[15] J. Fish, Q. Yu, and K. Shek. Computational damage mechanics for composite materials based on mathematical homogenization. International Journal for Numerical Methods in Engineering, 45(11):1657-1679, 1999.

[16] J. Fish and K. Shek. Multiscale analysis of large-scale nonlinear structures and materials. International Journal for Computational Civil and Structural Engineering, 1(1):79-90, 2000 .

[17] M. Šejnoha, R. Valenta, and J. Zeman. Nonlinear viscoelastic analysis of statistically homogeneous random composites. International Journal for Multiscale Computational Engineering, 2(4):645-674, 2004.

[18] J. Šejnoha, M. Šejnoha, J. Zeman, J. Sýkora, and J. Vorel. A mesoscopic study on historic masonry. preprint available at http://mech.fsv.cvut.cz/ zemanj/preprints/sem_07_ SeSeZeSyVo.pdf, 2007.

[19] TORAYCA. Technical data sheet, Toroyca T800H. Toray Carbon Fibers America, http: //www. torayusa.com.

[20] C. W. Ohlhorst. Thermal Conductivity Database of Various Strutural Carbon Carbon Composite Materials. NASA Technical Memorandum 4787, Lanley Research Center, Hampton, Virginia, 1997.

[21] G. Savage. Carbon-carbon composites. Chapman \& Hall, London, 1993.

[22] E. Fitzer and L. M. Manocha. Carbon reinforcement and Carbon-Carbon Composites. Springer verlag, Berlin, 1998.

[23] B. Tomková. Study of porous structure of C/C composites. In International Conference ICAPM, pages 379-387. Evora, Portugal, 2004.

[24] B. Tomková and B. Košková. The porosity of plain weave C/C composite as an input parameter for evaluation of material properties. In International Conference Carbon 2004, page 50. Providence, USA, 2004.

[25] LIM. System Lucia G, User guide. LUCIA - Laboratory Universal Computer Image Analysis, http://www.laboratory-imaging.com.

[26] J. L. Teplý and G. J. Dvorak. Bound on overall instantaneous properties of elastic-plastic composites. Journal of the Mechanics and Physics of Solids, 36(1):29-58, 1988.

[27] J.L. Auriault. Effective macroscopic description for heat conduction in periodic composites. International Journal of Heat and Mass Transfer, 26(6):861-869, 1983. 
[28] Z. Bittnar and J. Šejnoha. Numerical methods in structural engineering. New York: ASCE Press and Thomas Telford, U.K., 1996.

[29] FAMLAB. User's guide and Introduction. Version 2.3, COMSOL AB, 2002.

[30] L. Kubičár, V. Boháč, and V. Vretenár. Transient methods for the measurement of thermophysical properties: The pulse transient method. High Temperatures - High Pressures, 34:505-514, 2002. 